\title{
Dynamique spatio-temporelle de la pourriture brune des cabosses de cacaoyer due à phytophthora sp. dans le département d'Aboisso (Cote d'Ivoire)
}

\author{
POHE Jean ${ }^{1}$, MIAN Jacques Dutron² et OKOU Staelle Florence Famisso ${ }^{3}$ \\ 1. Maître de Conférences à I'Institut National Polytechnique Félix Houphouët Boïgny, DFR Agriculture et Ressources \\ Animales, Laboratoire de Phytopathologie et de Biologie Végétales, BP 1313 Yamoussoukro, Côte d'Ivoire, Auteur \\ correspondant: pohejean@yahoo.fr \\ 2. Ingénieur des Techniques Agricoles, EFPC de l'Institut National Polytechnique Félix Houphouët Boïgny de \\ Yamoussoukro \\ 3. Ingénieur des techniques Agricoles, Inspection phytosanitaire, Port Autonome d'Abidjan, Ministère de l'Agriculture. \\ Original submitted in on $19^{\text {th }}$ September 2019. Published online at www.m.elewa.org/journals/ on $31^{\text {st }}$ January 2020 \\ https://doi.org/10.35759/JABs.145.1
}

\section{RESUME}

Objectif : Cette étude a été menée pour évaluer le niveau de la pourriture brune, de suivre son évolution dans les plantations, au niveau des différentes parties du cacaoyer et de préciser l'emplacement des lésions sur les différentes cabosses infectées dans les cacaoyères dans le Département d'Aboisso Sudest de la Côte d'Ivoire.

Méthodologie et résultats : Cinq plantations ont été choisies avec dans chacune vingt plants mis en observation et sur lesquels trois parties ont été délimitées : le bas, le milieu et les branches. Sur chaque cabosse infectée la lésion est soit, proximale, latérale ou distale. Le dispositif est donc en blocs de Fischer avec un arrangement factoriel, 2 facteurs (localisation des cabosses dans l'arbre et la position des lésions sur les cabosse) avec chacun 3 niveaux soit, 9 traitements avec 5 répétions. L'analyse des taux des affections à Phytophthora donne une différence hautement significative d'une part entre les localisations des cabosses dans l'arbre et d'autre part, entre les positions des lésions sur les cabosses. Aussi, la position des lésions sur les cabosses diffère-telle selon la localisation de celles-ci dans l'arbre ( $p$-value $<1 \%$ ).

Conclusion: La pourriture brune sévit de façon endémique dans le département d'Aboisso. Les cabosses sur le tronc, notamment celles qui sont vers le bas, s'avèrent les plus vulnérables. Les lésions distales sont préférentiellement situées sur les cabosses vers le bas du tronc, alors que les proximales sont en grand nombre sur le milieu du tronc et les branches. Les Phytophthora étant des organismes telluriques, l'importance de leurs attaques sur les cabosses et sur les parties de celles-ci proches du sol, source d'inoculum primaire s'avérerait inévitable.

Application actuelle et potentielle des résultats: A partir de ces résultats, il convient de recommander d'éviter l'abandon de la protection avec des fongicides appropriés de la cacaoyère de cette localité comme ont tendance à le faire certains planteurs. Aussi, les applications de fongicides, surtout ceux de contact, par atomisation ne doivent-ils pas être dirigées seulement dans les branches mais également sur le tronc et surtout vers le bas des pieds des arbres.

Mots clés : pourriture brune, dynamique, cacaoyer, Aboisso, Côte d'Ivoire 


\section{ABSTRACT \\ Spatio-temporal dynamics of the brown rot of cocoa pots due to phytophthora sp. in the department of Aboisso (Cote d'Ivoire)}

Objective: This study was conducted to evaluate the level of brown rot, monitor its evolution in plantations, at the level of different parts of the cocoa tree and to specify the location of the lesions on the different pods infected in the cocoa farms in the Department. of Aboisso South-east of Ivory Coast.

Methodology and results: Five plantations were selected, each of which had twenty plants under observation and on which three parts were delineated: the bottom, the middle and the branches. On each infected pod the lesion is proximal, lateral or distal. The device is therefore in Fischer blocks with a factorial arrangement, 2 factors (location of the pods in the tree and the position of the lesions on the pod) with each 3 levels, i.e. 9 treatments with 5 repetitions. The analysis of the Phytophthora disease rates gives a highly significant difference between the location of the pods in the tree and between the positions of the lesions on the pods. Also, the position of the lesions on the pods differs according to the location of these in the tree ( $p$-value $<1 \%)$.

Conclusion: Brown rot is endemic in the Aboisso department. Pods on the trunk, especially those that are down, are the most vulnerable. The distal lesions are preferentially located on the pods down the trunk, while the proximal ones are in large numbers on the middle of the trunk and branches. Phytophthora being telluric organisms, the importance of their attacks on pods and parts of them that are close to the soil, a source of primary inoculum, would be inevitable.

Current and Potential Application of Results: From these results, it is advisable to avoid abandoning the protection with appropriate fungicides of the cocoa farm in this locality, as some planters tend to do. Also, the applications of fungicides, especially those of contact, by atomization should not be directed only in the branches but also on the trunk and especially down the feet of the trees.

Keywords: brown rot, dynamics, cocoa, Aboisso, Ivory Coast

\section{INTRODUCTION}

La Côte d'Ivoire est le premier pays producteur de fèves de cacao avec une production annuelle d'environ 1.700 .000 tonnes, soit 35 à $40 \%$ des exportations mondiales (ICCO, 2016). Sur le plan macroéconomique, l'économie cacaoyère fournit environ $40 \%$ de recettes d'exportation et contribue pour $10 \%$ à la formation du Produit Intérieur Brut (PIB). Au plan social, ce sont environ 600.000 chefs d'exploitations qui animent l'appareil de production, faisant ainsi vivre environ 6.000 .000 de personnes des revenus du cacao (ICRAF, 2011). L'on peut alors indiquer d'une part que la Côte d'Ivoire occupe une place prépondérante sur le marché international du cacao, et d'autre part que la cacao-culture revêt une importance cruciale pour son développement socio-économique. La conjugaison de ces deux états de fait rend le pays particulièrement sensible à l'analyse et à la bonne gestion des enjeux stratégiques inhérents à la durabilité de la production cacaoyère (Affou, 1993). Aussi, un des enjeux stratégiques d'une cacao-culture durable est-il son état sanitaire. Cependant, la pression parasitaire imposée par l'émergence de la pourriture brune des cabosses due au Phytophthora sp. Ces dernières années risque de compromettre la production cacaoyère en Côte d'lvoire et par conséquent perturber sa position de leader et sa stabilité socioéconomique. En effet, aujourd'hui en Côte d'Ivoire, la pourriture brune est devenue une maladie de grande ampleur, la perte de production qui était évaluée de 10 à $25 \%$ est passée à plus de $45 \%$ (Kouamé, 2006). L'émergence de la pourriture brune fut observée autour de 1999 dans la zone sud-est, à la frontière avec le Ghana (Koné, 1999 ; Pohé et al. 2013). Elle s'est rapidement généralisée dans toutes les zones productrices de cacao. Selon les études récentes, cette émergence serait accompagnée de la recomposition de la structure des populations de Phytophthora avec l'apparition de Phytophthora megakarya, l'espèce la plus virulente (Koné, 
1999 ; Pohé et al. ,2013). La pourriture brune est donc devenue une préoccupation inquiétante pour la Côte d'Ivoire en général et pour les producteurs de cacao en particulier. Les solutions à y apporter passent nécessairement par une connaissance

\section{MATERIELS ET METHODES}

Site expérimental: L'essai a été installé dans des plantations villageoises autour de la ville d'Aboisso au sud-est de la Côte d'Ivoire à environ 120 km d'Abidjan. La zone est caractérisée par un climat de type forestier avec quatre saisons (deux saisons pluvieuses intercalées de deux sèches).

Matériel végétal : Le matériel végétal est constitué de cacaoyers dont l'âge varie entre 10 et 12 ans et qui approfondie de la maladie. L'étude de la Dynamique spatio-temporelle de la pourriture brune des cabosses de cacaoyer due à Phytophthora sp, en Côte d'Ivoire, cas du département d'Aboisso en est une contribution.

n'ont font l'objet d'aucune application fongicide il y au moins deux ans.

Méthodes: Le dispositif expérimental: le dispositif adopté est celui de blocs de Fischer avec un arrangement factoriel des traitements comprenant 2 facteurs (Localisation des cabosses dans l'arbre et la Position des lésions sur la cabosse) avec chacun 3 niveaux (Haut, Milieu, Bas du cacaoyer) soit 9 traitements avec 5 répétions (Figure 1).

\begin{tabular}{|c|c|c|c|c|}
\hline$H L$ & $H L$ & $\mathrm{BL}$ & $\mathrm{MD}$ \\
\hline $\mathrm{BP}$ & $\mathrm{ML}$ & $\mathrm{MP}$ & $\mathrm{HP}$ & $\mathrm{MD}$ \\
\hline $\mathrm{ML}$ & $\mathrm{BP}$ & $\mathrm{HD}$ & $\mathrm{BL}$ & $\mathrm{BD}$ \\
\hline $\mathrm{BL}$ & $\mathrm{HP}$ & $\mathrm{BD}$ & $\mathrm{BP}$ & $\mathrm{HP}$ \\
\hline $\mathrm{MP}$ & $\mathrm{MD}$ & $\mathrm{MD}$ & $\mathrm{HL}$ & $\mathrm{BL}$ \\
\hline $\mathrm{HD}$ & $\mathrm{BL}$ & $\mathrm{HP}$ & $\mathrm{BD}$ & $\mathrm{BP}$ \\
\hline $\mathrm{BD}$ & $\mathrm{HD}$ & $\mathrm{BP}$ & $\mathrm{MP}$ & $\mathrm{MP}$ \\
\hline $\mathrm{HP}$ & $\mathrm{MP}$ & $\mathrm{ML}$ & $\mathrm{HD}$ & $\mathrm{ML}$ \\
\hline $\mathrm{MD}$ & $\mathrm{BD}$ & $\mathrm{HL}$ & $\mathrm{ML}$ & $\mathrm{HD}$ \\
\hline
\end{tabular}

Figure 1 : Schéma du dispositif expérimental

$H L$ : lésion latérale $(L)$ sur les cabosses du haut $(H)$ du cacaoyer

$H P$ : lésion proximale $(P)$ sur les cabosses du haut $(H)$ du cacaoyer

$H D$ : lésion distale (D) sur les cabosses du haut( $H)$ du cacaoyer

$B L$ : lésion latérale $(L)$ sur les cabosses du bas $(B)$ du cacaoyer

$B P$ : lésion proximale $(P)$ sur les cabosses du bas $(B)$ du cacaoyer

$B D$ : lésion distale $(D)$ sur les cabosses du bas(B) du cacaoyer

$M L$ : lésion latérale $(L)$ sur les cabosses du milieu $(M)$ du tronc du cacaoyer

$M P$ : lésion proximale( $(P)$ sur les cabosses du milieu $(M)$ du tronc du cacaoyer

$M D$ : lésion distale(D) sur les cabosses du milieu (M) du tronc du cacaoyer

Méthodologie : la méthodologie appliquée consiste à choisir cinq plantations autour de la ville d'Aboisso. Dans chaque plantation ont été repérés pour les observations vingt cacaoyers. Chaque cacaoyer est subdivisé en trois parties susmentionnées (figure 2). Ainsi chaque plantation constitue un bloc et comprend 20 cacaoyers à observer soit, 100 cacaoyers à observer. 


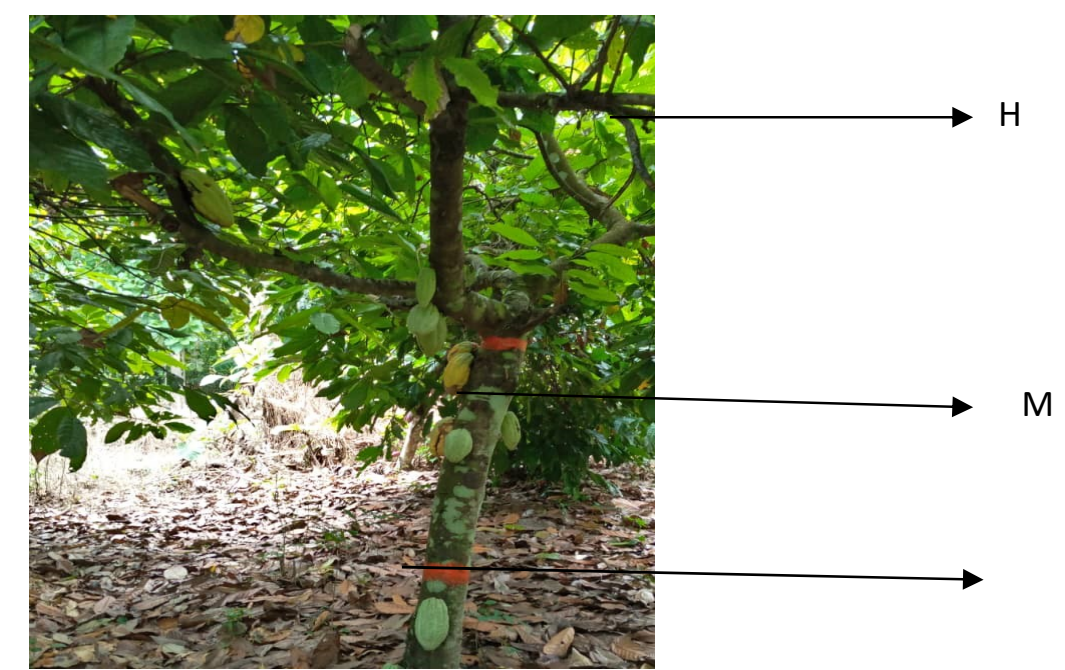

Figure 2 : Cacaoyer d'observation subdivisé en trois parties

Paramètres observés.

Les observations hebdomadaires ont porté sur :

-- Le nombre de cabosses nouvellement atteintes sur la base des symptômes de pourriture brune déjà décrits (Babacauh, 1980 ; Sonii, 2005 ; Fossouo, 2006 ; Pohe et al. 2013)

-- nombre total de cabosses de cacaoyer saines comme affectées observées

Analyses des données: Les niveaux des infections en taux des cabosses affectées sont calculés en

\section{RESULTATS}

Répartitions des cabosses observées dans les cacaoyers: La répartition des cabosses observées dans les différentes parties du cacaoyer est présentée dans le graphique de la figure 3. Sur 6260 cabosses observées dans l'ensemble il y a : pourcentage des cabosses affectées par la pourriture brune par rapport au total des cabosses observées. Les courbes de l'évolution des taux hebdomadaires de la maladie dans le temps ont été établies et ont permis de suivre l'évolution du niveau de la maladie en fonction des différents traitements. La comparaison des moyennes portant sur les taux des attaques cumulées avec ANOVA et le test de comparaison multiple de Duncan à l'aide du logiciel SPSS, a servi à évaluer et à comparer les différents traitements.

2124 cabosses observées au bas des pieds du cacaoyer (B) ;

2575 dans la partie médiane (M) ;

1561 au niveau des branches 


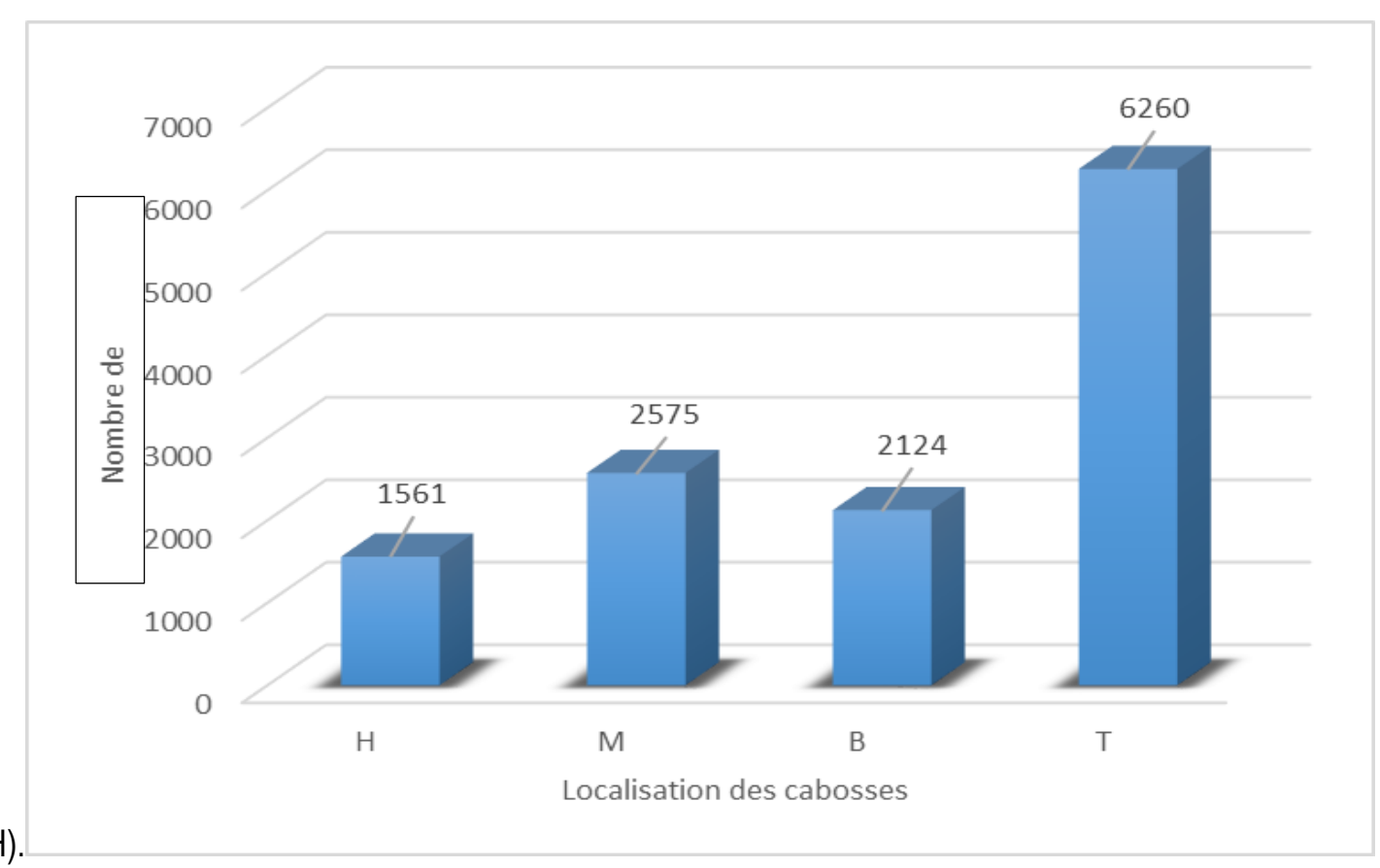

Figure 3 : Répartition des cabosses observées dans l'arbre

L'analyse de variance (tableau 1) portant sur ces données relatives à la répartition des cabosses dans les cacaoyers, montre une différence significative entre le nombre de cabosses observées en passant d'une partie de l'arbre à l'autre.

Tableau 1: Analyse de variance portant sur la répartition dans l'arbre des nombres de cabosses observées

\begin{tabular}{|l|c|c|c|c|c|}
\hline & Somme des carrés & Ddl & Moyenne des carrés & F & Signification \\
\hline Inter-groupes & 103237,733 & 2 & 51618,867 & 27,462 & 0,000 \\
Intra-groupes & 22555,600 & 12 & 1879,633 & & \\
Total & 125793,333 & 14 & & & \\
\hline
\end{tabular}

Le test de comparaison multiple de DUNCAN effectué à cet effet (tableau 2), montre trois groupes :

Groupe 1 : le groupe 1 est constitué des cabosses issues des branches, en nombre moins élevé que celles des deux groupes 2 et 3.

Groupe 2: le groupe 2 est constitue des cabosses du bas du tronc de l'arbre (B) en nombre moins élevés que celles du groupe 3 et en nombre plus élevé que celles du groupe 1.

Groupe 3 : le groupe 3 est constitué des cabosses du milieu du tronc en nombre élevé que celles des deux premiers groupes.

Tableau 2 : Test de comparaison multiple de DUNCAN sur le nombre des cabosses observées selon les parties du cacaoyer.

\begin{tabular}{|l|c|c|c|c|}
\hline NIVEAU & N & \multicolumn{3}{|c|}{ Sous-ensemble pur alpha $=\mathbf{0 . 0 5}$} \\
\cline { 3 - 5 } & & $\mathbf{1}$ & $\mathbf{2}$ & $\mathbf{3}$ \\
\hline Haut de l'arbre & 5 & 312.2000 & & \\
Bas de l'arbre & 5 & & 424.8000 & \\
Milieu de l'arbre & 5 & & & 515.0000 \\
Significatif & & 1,000 & 1,000 & 1,000 \\
\hline
\end{tabular}




\section{Importance de la pourriture brune}

Evolution de la pourriture brune : Les courbes issues des résultats des observations hebdomadaires sont représentées dans le graphique de la figure 4 : Les différentes courbes présentent la même allure caractéristique de l'évolution de la pourriture brune des cabosses de cacaoyer, avec des pics par endroit comme l'ont déjà décrite certains auteurs (Brassier and Hansen, 1992 ; Pohe, 1996, 2012 ; Pohé et al. 2013). Cependant, il apparait une différence entre les niveaux des infections en fonction de l'emplacement des cabosses dans l'arbre. L'importance des infections apparait diminuer en partant du bas vers le haut du tronc des cacaoyers.

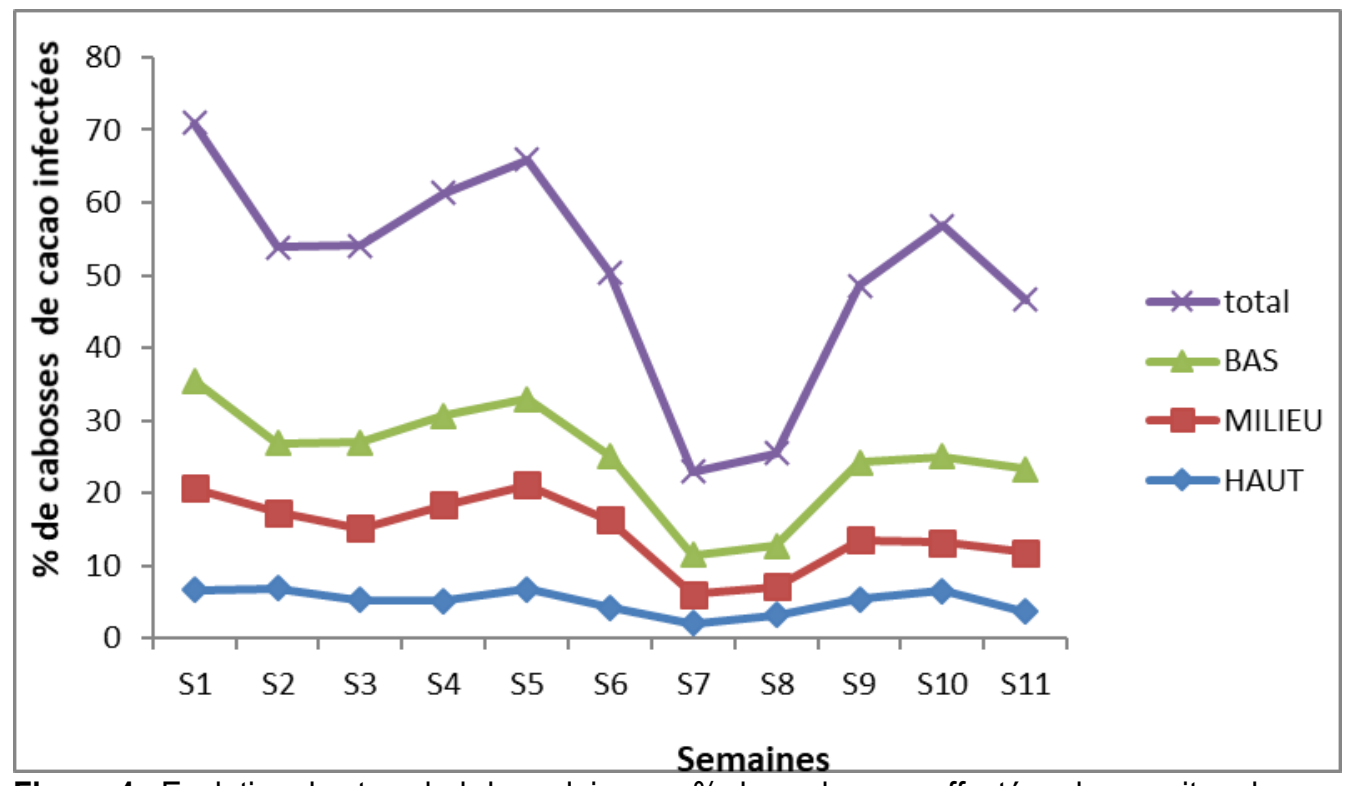

Figure 4 : Evolution des taux hebdomadaires en $\%$ des cabosses affectées de pourriture brune

Comparaison des taux cumulés de cabosses infectées

Taux cumulés des cabosses infectées: Les taux moyens des attaques cumulées de la pourriture brune sont dans l'ordre de 25,47 à $28,9 \%$ pour l'ensemble des parcelles. Ils varient de 4,87 à 5,67 dans les branches, de 9,29 à 10,42 au milieu du tronc et de 11,03 à 13,27 au bas du tronc de l'arbre (Tableau 3).

Tableau 3: Taux cumulés en $\%$ de cabosses affectées dans les parcelles selon la partie de l'arbre

\begin{tabular}{|l|c|c|c|c|c|}
\hline & $\mathrm{R} 1$ & $\mathrm{R} 2$ & $\mathrm{R} 3$ & $\mathrm{R} 4$ & $\mathrm{R} 5$ \\
\hline haut & $4,87 \%$ & $5,21 \%$ & $5,16 \%$ & $5,15 \%$ & $5,67 \%$ \\
\hline milieu & $10,42 \%$ & $10,42 \%$ & $10,08 \%$ & $9,29 \%$ & $10,15 \%$ \\
\hline bas & $12,93 \%$ & 13,27 & $11,49 \%$ & $11,03 \%$ & $11,44 \%$ \\
\hline Total & 28,22 & 28,9 & 26,73 & 25,47 & 27,26 \\
\hline
\end{tabular}

Position des lésions sur les cabosses: Les différentes positions des lésions sur les cabosses observées en plantation sont représentées dans la figure 5. Elles sont latérales, proximales et distales. 


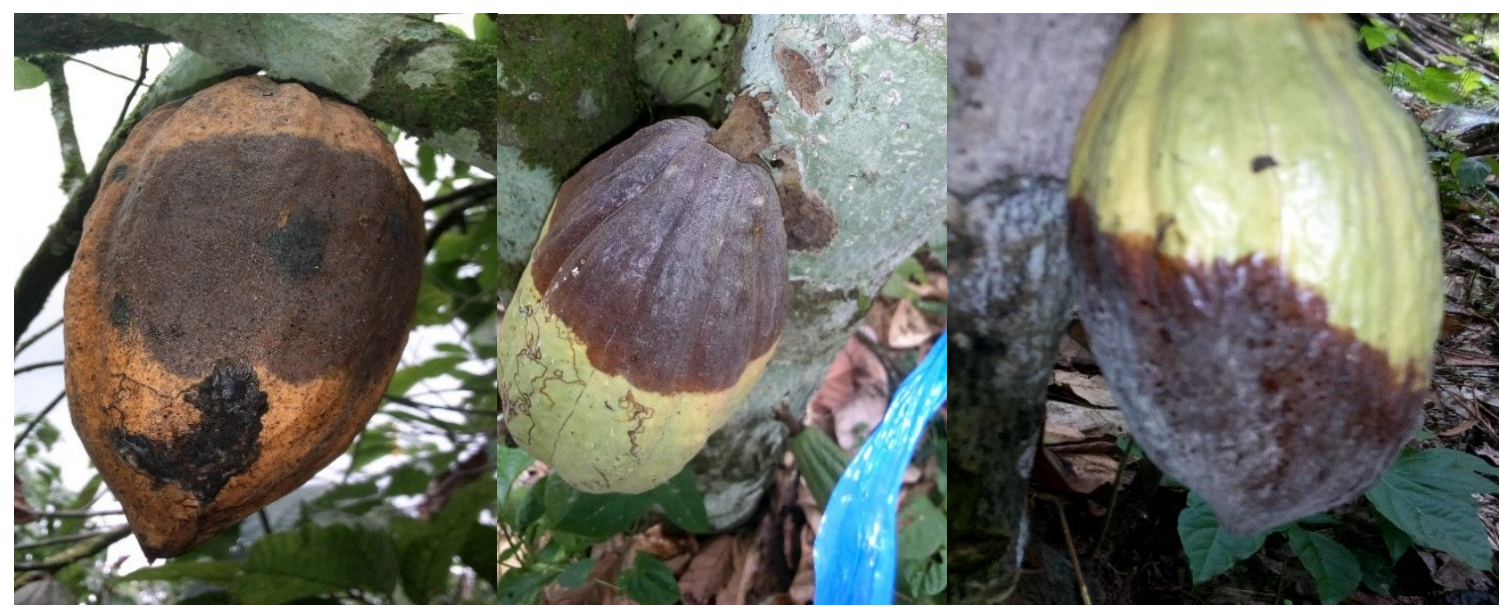

Figure 5: Différentes positions des lésions sur les cabosses

Analyse de variance: Les résultats globaux portant sur les affections de pourriture brune en fonction de la position de la lésion sur la cabosse et l'emplacement de celle-ci dans l'arbre sont consignés dans le tableau 4.

Tableau 4. Taux des différentes lésions en fonction de l'emplacement des cabosses dans l'arbre

\begin{tabular}{|l|c|c|c|c|c|}
\hline & R1 & R2 & R3 & R4 & R5 \\
\hline HP & $2,20 \%$ & $2,36 \%$ & $2,58 \%$ & $2,03 \%$ & $2,32 \%$ \\
\hline HL & $0,91 \%$ & $0,97 \%$ & $1,40 \%$ & $1,01 \%$ & $1,29 \%$ \\
\hline HD & $1,75 \%$ & $1,87 \%$ & $1,17 \%$ & $2,10 \%$ & $2,06 \%$ \\
\hline MP & $4,49 \%$ & $4,88 \%$ & $4,30 \%$ & $4,06 \%$ & $4,99 \%$ \\
\hline ML & $1,59 \%$ & $1,95 \%$ & $2,11 \%$ & $2,61 \%$ & $2,32 \%$ \\
\hline PD & $3,88 \%$ & $3,58 \%$ & $3,67 \%$ & $2,61 \%$ & $2,83 \%$ \\
\hline BP & $4,26 \%$ & $4,23 \%$ & $3,67 \%$ & $3,92 \%$ & $3,87 \%$ \\
\hline BL & $1,97 \%$ & $2,60 \%$ & $2,11 \%$ & $1,96 \%$ & $1,97 \%$ \\
\hline BD & $6,69 \%$ & $6,43 \%$ & $5,70 \%$ & $5,15 \%$ & $5,59 \%$ \\
\hline
\end{tabular}

L'analyse de variance au tableau 5 , portant sur ces résultats relatifs aux taux de cabosses infectées selon les 2 facteurs (positions et localisation) et les 3 niveaux $(H, M, B)$ consignés dans le tableau 5 , montre qu'il n'y a pas de différence significative entre les blocs que constituent les plantations ( $p$-value $>0,05$ ). Cependant, il y a une différence hautement significative entre les différentes localisations dans l'arbre et une différence hautement significative entres les positions des lésions sur les cabosses. Aussi, l'interaction localisation des cabosses dans l'arbre et la position des lésions sur les cabosses estelle significative, en d'autres termes les positions des lésions sur les cabosses diffèrent de façon hautement significative selon la localisation de celles-ci dans l'arbre ( $p$-value $<1 \%$ ).

Tableau 5 : Analyse de variance portant sur les données des 2 facteurs avec 3 niveaux

\begin{tabular}{|l|c|c|c|c|c|}
\hline \multicolumn{1}{|c|}{ Sources de variation } & ddl & SC & CM & F & P-value \\
\hline Bloc & 4 & 0,709 & 0,177 & 1,181 & 0,338 \\
\hline Localisation & 2 & 40,815 & 20,408 & 135,895 & 0,000 \\
\hline Position & 2 & 34,500 & 17,250 & 114,869 & 0,000 \\
\hline Localisation*Position & 4 & 19,667 & 4,917 & 32,741 & 0,000 \\
\hline Résiduelle & 32 & 4,806 & 0,150 & & \\
\hline Totale & 44 & 100,497 & & & \\
\hline
\end{tabular}

** : Hautement significatif $P=0,000<1 \%$

- $\quad$ comparaison des moyennes des taux cumulés des cabosses infectées

- $\quad$ Localisation des cabosses affectées dans l'arbre 
Le test de comparaison multiple de Duncan effectué sur les moyennes des taux des cabosses affectées selon leur localisation dans l'arbre (tableau 6) montre trois groupes.

-Groupe 1 : le groupe 1 est composé uniquement des cabosses des branches $(\mathrm{H})$ dont les taux d'infection sont inférieurs à ceux de celles des deux groupes 2 et 3 ;
-Groupe 2 : le groupe 2 est composé des cabosses du milieu du tronc (M) dont les taux d'infection sont inférieurs à ceux de celles du groupe 3 et supérieurs à ceux de celles du groupe1;

Groupe 3: le groupe 3 est constitué des cabosses affectées du bas du tronc (B) dont les taux sont supérieurs à ceux de celles des deux premiers groupes.

Tableau 6 : Comparaison des moyennes des taux des cabosses infectées selon leur emplacement dans les cacaoyers

\begin{tabular}{|c|c|c|c|c|}
\hline \multirow[t]{2}{*}{ Niveau } & \multirow[t]{2}{*}{$\mathrm{N}$} & \multicolumn{3}{|c|}{ Sous-ensemble pour alpha $=0.05$} \\
\hline & & 1 & 2 & 3 \\
\hline $\begin{array}{l}\text { Haut du cacaoyer } \\
\text { Milieu du cacaoyer } \\
\text { Bas du cacaoyer }\end{array}$ & $\begin{array}{l}5 \\
5 \\
5\end{array}$ & 5.2120 & 10.0720 & 12.0320 \\
\hline Signification & & 1,000 & 1,000 & 1,000 \\
\hline
\end{tabular}

Position des lésions: La différence des taux des cabosses infectées selon la position des lésions sur la cabosse est significative selon ANOVA (tableau 7). Cependant, le test de comparaison multiple de Duncan effectué à cet effet (Tableau 8) montre seulement deux groupes:
Groupe 1: le groupe 1 est composé uniquement des lésions latérales dont les taux sont inférieurs à ceux de celles du groupe2.

Groupe 2: le groupe 2 comprend les cabosses de lésions proximales et distales de taux égaux entre eux et supérieurs à ceux de celles du groupe1.

Tableau7 : Comparaison des moyennes des taux des cabosses selon la position des lésions dans l'ensemble

\begin{tabular}{|l|c|c|c|}
\hline Lésion & $N$ & \multicolumn{2}{|c|}{ Sous-ensemble pour alpha =0.05 } \\
\cline { 3 - 4 } & & 1 & 2 \\
\hline Latérale & 5 & 5.3640 & \\
Proximale & 5 & & 10.8720 \\
Distale & 5 & & 11.0220 \\
Signification & & 1,000 & 0,953 \\
\hline
\end{tabular}

$\begin{array}{lrrr}\text { Classement des taux d'attaques : } & \text { DISTALE } & = & \text { PROXIMALE } \\ 11,0220 & \text { LATERALE } \\ 10,8720 & 5,3640\end{array}$

Interaction localisation des cabosses- Position des lésions sur les cabosses : L'interaction Localisation-
Position étant significative (Tableau 6) il va alors faire une analyse par niveau de localisation. 
Haut

Tableau 8 : Comparaison des moyennes des taux des lésions au niveau Haut de l'arbre

\begin{tabular}{|c|c|c|c|c|}
\hline Position & $N$ & \multicolumn{3}{|c|}{ Sous-ensemble pour alpha =0.05 } \\
\cline { 3 - 5 } & & 1 & 2 & 3 \\
\hline L & 5 & 1,116 & & \\
$D$ & 5 & & 1,790 & 2,298 \\
P & 5 & & & 1,000 \\
\hline Signification & & 1,000 & 1,000 & \\
\hline
\end{tabular}

Au niveau de la localisation haute du cacaoyer, la $(1,116 \%)$, les pourcentages étant tous différents les uns position proximale a le plus grand taux $(2,298 \%)$ suivi des autres.

de la position distale $(1,79 \%)$ et de la position latérale

- $\quad$ Milieu du cacaoyer

Tableau 9 : Comparaison des moyennes des taux des lésions au niveau milieu de l'arbre

\begin{tabular}{|c|c|c|c|c|}
\hline \multirow[t]{2}{*}{ Position } & \multirow[t]{2}{*}{$\mathrm{N}$} & \multicolumn{3}{|c|}{ Sous-ensemble pour alpha $=0.05$} \\
\hline & & 1 & 2 & 3 \\
\hline \begin{tabular}{|l} 
\\
$D$ \\
$P$ \\
Signification
\end{tabular} & $\begin{array}{l}5 \\
5 \\
5\end{array}$ & 2,1160 & $\begin{array}{l}3,3140 \\
1,000\end{array}$ & $\begin{array}{l}4,5440 \\
1,000\end{array}$ \\
\hline
\end{tabular}

Au niveau de localisation milieu du cacaoyer, la position proximale a le plus grand taux $(4,544 \%)$ suivie de la position distale $(3,314 \%)$ et de la position latérale

(2,116\%), les pourcentages étant tous différents les uns des autres.

- $\quad$ Bas du cacaoyer

Tableau 10: Comparaison des moyennes des taux des lésions au niveau bas de l'arbre

\begin{tabular}{|l|l|l|l|l|}
\hline Position & N & \multicolumn{3}{|c|}{ Sous-ensemble pour alpha $=0.05$} \\
\cline { 2 - 5 } & & 1 & 2 & 3 \\
\hline L & 5 & 2,1220 & & \\
P & 5 & & 3,9900 & \\
Signification & 5 & 1,000 & 1,000 & 5,9120 \\
\hline
\end{tabular}

Au niveau de localisation basse du cacaoyer, la position Distale a le plus grand taux $(5,912 \%)$ suivi de la position proximale $(3,99 \%)$ et de la position latérale

\section{DISCUSSION ET CONCLUSION}

Niveau de la maladie : II ressort de nos observations, que la pourriture brune avec $28,22 \%$ de cabosses de cacao infectées, malgré les récoltes sanitaires régulières, sévit de façon endémique dans le département d'Aboisso. Quant à son évolution dans le temps, dans l'ensemble, les courbes y afférentes suivent une allure caractéristique connue des infections à Phytophtora sp., Les pics par endroit correspondant aux taux les plus élevés de la maladie seraient étroitement liés aux pluies qui favoriseraient leur
$(2,116 \%)$. Les pourcentages moyens sont tous différents les uns des autres.

installation et leur développement (Duniway, 1979; Babacauh, 1980; Brasier et Hansen, 1992; Pohé, 1996 ; Pohé et al., 2013).

Importance de la maladie selon l'emplacement des cabosses dans l'arbre: Les taux de la pourriture brune observés sous nos conditions expérimentales indiquent bien que les branches, constituant la partie haute du cacaoyer où moins de cabosses ont été observées présentent moins de cabosses affectées par la pourriture brune alors que les affections sont 
importantes dans la parties du milieu et du bas de l'arbre qui contiennent plus de fruits. Un tel phénomène est connu en épidémiologie, l'intensité de la maladie diminue avec la réduction de la population cible (Zadoks et Schein, 1976 ; Pohé, 1996). Aussi, la source d'inoculum jouerait-elle un rôle important et qui expliquerait l'abondance des attaques vers le bas de l'arbre. Pendant la phase épidémique de contamination, les attaques commenceraient, en premier lieu, sur les cabosses qui sont proches du sol, source de contamination. En effet, le rapprochement de la source d'inoculum et la cible est un facteur favorable au développement de la maladie (Savary et Zadoks, 1992 ; Pohé, 2013). Tarjot (1973) a montré que les premières cabosses infectées lors du déclenchement de l'attaque sont en général situées à proximité du sol ou celles ayant un contact direct avec le sol. Aussi, a til démontré que lors des pluies, l'effet « splash » produit par les gouttes de pluie projette les propagules du champignon contenus dans le sol sur les cabosses basses. Celles-ci sont alors contaminées. Phytophthora megakarya serait l'une des espèces de Phytophthora la plus transmissible par le sol selon le même auteur. Les coussinets floraux sont également à l'origine d'un nombre élevé d'infections. Phythophthora. Palmivora se transmettrait plus aisément par les coussinets que $P$. megakarya, car le premier a une plus grande aptitude à survivre à la jonction coussinetpédoncule (Brasier et al, 1981).

Position des lésions sur cabosses: Les lésions proximales et distales sont en nombre égal et sont plus importantes que les lésions latérales dans nos

\section{REFERENCES BIBLIOGRAPHIQUES}

Affou S.Y. 1993. The Impact of government Policy on Cocoa Production in C.I. Contribution à la conférence internationale organisée à Bali par CIRAD et ASKINDO du 19 au 22 Octobre 1993 sur le thème de l'économie cacaoyère, Bali Nusa Dua.

Babacauh, K.D., 1980. Structure et dynamique des populations de Phytophtora sp. parasite du cacaoyer (Theobroma cacao L.). Thèse Doctorat d'Etat, Université d'Orsay N² 2344. 180 Pages.

Babacauh k.d. 1982. Rôle des communautés d'insectes et de l'eau dans la dissémination de Phytophthora palmivora (Butl.) Bult. Emmend Bras.et Griff.dans les cacaoyères de Côte d'Ivoire Café Cacao Thé $2: 31-36$. observations. II est connu que les Phytophthora sont des organismes telluriques et ils se conservent dans le sol qui constituerait une source d'inoculum primaire (Pohé ,1996 ; 2012) et ceux inféodés au cacaoyer en plus du sol se conservent aussi dans les coussinets floraux (Blaha et latode 1976 ; Babacauh, 1982). Ces sites de conservation influenceraient les phases épidémiques de la pourriture brune ce qui conduirait à établir un lien entre les lésions et l'emplacement des cabosses dans l'arbre et par conséquent avec la source d'inoculum. En effet, les cabosses pendant sur le tronc du cacaoyer avec la partie distale tournée vers le bas en direction et proche du sol, source d'inoculum seraient prédisposées aux attaques des Phytophthora dans leur partie distale. II en est de même des lésions proximales qui seraient occasionnées par les propagules issues des coussinets floraux autres sources d'inoculum primaire (Brasier et al. 1981). Les lésions distales seraient donc préférentiellement occasionnées par l'inoculum venant du sol, les lésions proximales de l'inoculum des coussinets et les lésions latérales seraient réalisées grâce aux agents de dissémination des propagules. A partir de ces résultats, il conviendrait de recommander aux producteurs d'Aboisso de protéger les cacaoyers avec des fongicides appropriés car la pourriture brune sévit encore de façon endémique. Aussi, que les applications de fongicides, surtout ceux de contact, par atomisation ne soient pas dirigés seulement dans les branches mais également sur le tronc et surtout vers le bas du pied des arbres.

Blaha, G. And R. Latode, 1976. The primary criteria for selection of cacao in Cameroun: The resistance to pod rot losses: Variations in reactions and development of lesions due to ecological and physiology of fruits. Cafe Cacao, 20: 97-116.

Brasier C. M., Griffin M J., And Maddison A.C., 1981. The cocoa black pod Phytophthora; in epidemiology of Phytophthora on cacao in Nigeria. Edited by GREGORY, .P.H and MADDISON A.C. PP 18-30.

Brasier, C.M. And Hansen, E.M.1992. Evolutionary Biology of Phytophthora. Part.l I : phylogeny, speciation, and population structure. Annu. Rev. Phytopathology 30, PP 173-200.

Duniway, J.M. 1979. Water relations of water molds. Annu. Rev. Phytopathology 17, PP 395-341 
Fossouo K.A.L., 2006. Développement spatio-temporel de la pourriture brune des fruits du cacaoyer : cas de deux parcelles paysannes des provinces du centre et sud Cameroun. Mémoire pour l'obtention du Master en Statistique Appliquée. Université de Yaoundé I, Ecole Nationale Supérieure Polytechnique, Département de mathématiques et de sciences physiques. Page 93.

ICCO, 2011. International Cocoa and Coffee Organization, Annual report. P 42.

ICRAF, 2011. Presentation du projet Vision For Change. p.10.

Kone .Y.R. 1999. Etude de la structure actuelle des populations de Phytophtora sp. Agents de la pourriture brune des cabosses du cacaoyer (Theobroma cacao L.) en Côte d'Ivoire. Mémoire en vue de l'obtention du Diplôme d'Agronomie Approfondie, option défense des cultures, ESA Yamoussoukro. 111 Pages.

Kouame K.D., 2006. Structure et dynamique des populations de Phytophtora sp., agent de la pourriture brune des cabosses de cacaoyer (Theobroma cacao L) en Côte d'lvoire. Mémoire de DEA. UFR Biosciences. Université de Cocody, Abidjan, côte d'Ivoire. p74.

Pohe J., 1996. Contribution à l'étude de la pourriture du cœur et des noix immatures du cocotier dues à Phytophthora katsurae (KO et CHANG) en Côte d'Ivoire. Thèse de doctorat de l'Institut National Agronomique Paris-Grignon, France. P179.

Pohe J, 2012. Phytophthora spp. agents des maladies de diverses plantes. Rev. Ivoir. Sci. Technol. 19 (2012) 72-87 ISSN 1813-3290 http://www.revist.ci.

Pohe J, 2013. Sources d'inoculum primaire de Phytophthora katsurae (Ko et Chang) agent de la pourriture du cœur et des noix immatures du cocotier en Côte d'Ivoire. Journal of Animal and plant sciences, 16(3): PP23551-2361 ISSN 2071-7024 http : //www.m.elewa.org

Pohe J, Koula J, Rabe G. R, Dezai. L, 2013. Agressivité de la pourriture brune des cabosses de cacaoyer dans le sud-est de la Côte d'Ivoire. Journal of Animal and Plant Sciences. 20 (2): 3126-3136 ISSN 2071-7024 https://m.elewa.org/Journals/about-japs/

Savary S et Zadoks J.C, 1992. Analysis of crop loss in the multiple pathosystem groundnut-rust-late leaf spot. II. Study of the interactions between diseases and crop intensification in factorial experiments. Crop protection 11(2) : PP110120.

Sonii D., 2005. A la découverte de la production durable du cacao: guide de formation participative des planteurs. La lutte intégrée. Sustainable Tree Crops Program, International Institute of Tropical Agriculture Yaoundé, Cameroun. p 45.

Tarjot. M, 1973. Contribution à l'étude du comportement des feuilles de cacaoyer envers le Phytophthora palmivora. Café-Cacao-Thé 16 (4) PP333-338.

Zadocks J.C et Schein R.D., 1976: The role of crop populations and their development, illustrated by means of a simulator. Epimul.76 Annals of the New York Academy of Science, 287, PP164-190 